\title{
Propiedades psicométricas del Cuestionario de Evaluación del Conocimiento sobre Estrategias de Autorregulación en universitarios (CEA-U)
}

\author{
Pedro Rosário', José Carlos Núñez ${ }^{\star}$, Rebeca Cerezo², Estrella Fernández², \\ Paula Solano ${ }^{2}$ y Natalia Amieiro ${ }^{2}$ \\ 'Universidad do Minho \\ 2Universidad de Oviedo
}

\begin{abstract}
Resumen: Se dispone de suficientes instrumentos de evaluación sobre el uso de estrategias de aprendizaje autorregulado por parte de los estudiantes de las diferentes etapas educativas. Sin embargo, no existen instrumentos fiables y válidos que aporten información sobre el grado de conocimiento del estudiante sobre lo que implica un comportamiento autorregulado a la hora de aprender, a pesar de que el nivel del conocimiento previo es un determinante importante de la conducta posterior. Por ello, el objetivo de este estudio fue el análisis de las propiedades psicométricas del Cuestionario de Conocimiento Autorregulatorio (CEA) en estudiantes universitarios (CEA-U). En base a un diseño de medidas repetidas (dos medidas con un intervalo temporal de seis meses) se ha aplicado el instrumento de evaluación a una amplia muestra de estudiantes de las titulaciones de psicología y educación. La validez y la fiabilidad de la escala se estudió por una doble vía: la validez estructural y la predictiva; así como la fiabilidad por consistencia interna y test-retest. Los resultados obtenidos muestran al CEA-U como un instrumento válido y fiable para su utilización con estudiantes universitarios.
\end{abstract}

Palabras clave: Autorregulación del aprendizaje, Conocimiento, Educación Superior, Propiedades psicométricas.

\section{Psychometric properties of the Knowledge Evaluation Questionnaire on self-regulation strategies} in university students (CEA-U)

Abstract: There are enough assessment instruments available on the use of self-regulated learning strategies by the students of the different educational stages. However, there are no reliable and valid instruments that provide information on the student's level of knowledge about what self-regulated behavior implies when it comes to learning. However, it has repeatedly been proven that the level of prior knowledge is an important determinant of subsequent behavior. Therefore, the aim of this study was the analysis of the psychometric properties of the Self-Regulatory Knowledge Questionnaire (CEA) in university students (CEA-U). Based on a design of repeated measures (two measures with a time interval of six months) the instrument has been applied to a wide sample of students of psychology and education degrees. The validity and reliability of the scale was studied in two ways: structural and predictive validity; as well as reliability for internal consistency and test-retest. The results obtained show the CEA-U as a valid and reliable instrument for use with university students.

Keywords: Self-regulation of learning, Knowledge, Higher Education, Psychometric properties.

Desde hace ya algunos años existe una gran preocupación por la calidad de los

Recibido: 21/01/2019 - Aceptado: 24/02/2019 - Avance online: 02/04/2019

*Correspondencia: José Carlos Núñez.

Universidad de Oviedo, Oviedo, España.

C.P: 33003, Oviedo, España.

E-mail: jcarlosn@uniovi.es

Rosário, P., Núñez, J.C., Cerezo, R., Fernández, E., Solano, P. y Amieiro, N. (Avance Online).Propiedades psicométricas del Cuestionario de Evaluación del Conocimiento sobre Estrategias de Autorregulación en universitarios (CEA-U). Revista de Psicología Educación, 14(2), 144-156, https://doi.org/10.23923/rpye2019.02.179 aprendizajes del estudiante universitario y, en general, por su formación integral (Allgood, Risko, Álvarez \& Fairbanks, 2000). Por un lado, se han estudiado factores relacionados con aspectos externos, como el tiempo dedicado al estudio (Plant, Ericsson, Hill \& Asberg, 2005), la realización de actividades extra 
(Cheung \& Kwok, 1998; Rosário, Mourao, Soares, Chaleta, Grácio, Simoes, Núñez \& González-Pienda, 2005) y la influencia de factores contextuales y ambientales (Pike, 2005). Por otro lado, también ha sido estudiada la relevancia de factores internos como, por ejemplo, variables de personalidad (Paunonen \& Ashton, 2001) o factores vinculados con su forma de aprender, como los enfoques de aprendizaje (Biggs, 2001; Entwistle \& Waterston, 1988), las estrategias de aprendizaje (Cano, 2005; Tuckman, 2003), o los aspectos motivacionales que le condicionan el inicio, sostén y dirección del proceso (Wolters, 2003).

Desde una perspectiva amplia, abarcando los factores más importantes señalados, muchos autores caracterizan al estudiante universitario con éxito como un "estudiante autorregulado" (Allgood et al. 2000; Williams \& Hellman, 1998, 2004; Garavalia \& Gredler, 2002; Pintrich \& DeGroot, 1990; Zimmerman, 1998; Zimmerman \& Bandura, 1994; Nota, Soresi \& Zimmerman, 2005). Los estudiantes autorregulados dirigen su aprendizaje a través de la puesta en práctica de una serie de estrategias cognitivas, metacognitivas, motivacionales y de apoyo que les permiten construir sus conocimientos de forma constructiva, siendo capaces de regular y controlar de forma intencional todo el proceso — conocen sus habilidades, los conocimientos que poseen, saben qué deben hacer para aprender, han aprendido a monitorizar sus conductas de estudio, ajustan sus conductas y actividades a las demandas de estudio, están motivados por aprender y son capaces de regular su motivación, etc. (Pintrich, 2000, 2004). Lo que claramente los identifica como gestores eficaces de sus aprendizaje no es tanto su utilización aislada de estrategias de aprendizaje, sino su iniciativa personal, su perseverancia en la tarea y las competencias exhibidas, independientemente del contexto en el que ocurre el aprendizaje. Los alumnos "auto-reguladores" se centran en su papel como agentes: son conscientes de que el éxito académico depende sobre todo de su actividad e implicación (Bandura, 2001; Zimmerman, 2002).
Por todo ello, la autorregulación de aprendizaje resulta fundamental de cara al éxito académico en los estudios superiores, ya que éste está muy relacionado tanto con la realización de un trabajo personal de intensa implicación en tiempo de estudio, como con el patrón estratégico de autorregulación utilizado (Rosário, et al., 2005). Por otro lado, la autorregulación del aprendizaje no solo influye en el rendimiento sino en otros aspectos fundamentales en la universidad como la constancia y la persistencia (Nota, Soresi \& Zimmerman, 2005), o las conductas de procrastinación (Cerezo, SánchezSantillán, Paule, \& Núñez, 2016).

El alumno universitario debería ser capaz de autorregular su aprendizaje y actividad de estudio, lo cual le permitiría ser autónomo a la hora de aprender significativa y constructivamente durante toda su vida, tal y como queda reflejado en las nuevas legislaciones recogidas en la Declaración de Bolonia y otros documentos europeos (González \& Wagenaar, 2003). También se hace hincapié en la necesidad de dotar al estudiante de una serie de competencias que los capaciten para enfrentarse a las nuevas demandas de nuestra sociedad caracterizada por los cambios en el conocimiento y la información. En definitiva, dotar al alumno de las competencias necesarias para aprender de forma autónoma se considera como uno de los grandes retos de en la nueva legislación europea.

Sin embargo, la situación real que encontramos en la universidad es que la mayoría de los estudiantes no están adecuadamente preparados para lo que se requiere de ellos en la universidad, no son alumnos autorregulados (Allgood et al. 2000). Por ello, a partir de todo este conocimiento que indica qué características deben poseer los estudiantes universitarios para tener éxito a la hora de estudiar y aprender, la cuestión más importante radica en saber qué saben y qué hacen realmente estos alumnos cuando afrontan sus tareas como estudiantes universitarios para, a partir de ahí, identificar carencias y planificar intervenciones (Allgood et al., 2000; Winnie \& Jamienson, 2002, 2003). La evaluación del conocimiento personal 
sobre SRL, en consecuencia, parece una tarea necesaria para la prevención de dificultades en los procesos de aprendizaje universitario.

La toma de conciencia de la relevancia de los procesos autorregulatorios en el aprendizaje académico, y para un aprendizaje autónomo, ha llevado al desarrollo de multitud de instrumentos de medida de SRL, o variables cercanas conceptualmente (e.g., estrategias de aprendizaje, procesos de estudio, procesos metacognitivos, estrategias SRL en dominios específicos, et., o combinación de constructos relacionados como, por ejemplo, estrategias motivacionales, cognitivas y afectivas) (Núñez, Amieiro, Álvarez, García \& Dobarro, 2015), principalmente de tipo autoinforme (e.g., Escala de Estrategias de Aprendizaje -ACRA-, Escala de Estrategias de Aprendizaje Contextualizado ESEAC-, Learning and Study Strategies Inventory -LASSI-, State Metacognitive Inventory -SMI-, Motivated Strategies for Learning Questionnaire -MSLQ-, Patterns of Adaptive Learning Scales -PALS-, Sistema Integrado e Interactivo de Evaluación de Atribuciones Causales y Procesos y Estrategias de Aprendizaje -SIACEPA-, SelfRegulation Strategy Inventory -SRSI-SR-, Escala de Evaluación de la Autorregulación del Aprendizaje a partir de Textos ARATEX-R). El autoinforme es el formato principalmente utilizado posiblemente debido a que es relativamente fácil de diseñar, administrar y puntuar (Winne \& Perry, 2000), y ello a pesar de los importantes inconvenientes observados con su utilización (Boekaerts \& Corno, 2005; Núñez, et al., 2006; Zimmernam, 2008).

Todos estos instrumentos aportan información sobre el uso de estrategias autorregulatorias. Sin embargo, no disponemos de instrumentos fiables y teóricamente validos que aporten información sobre el grado de conocimiento del estudiante sobre lo que implica un comportamiento autorregulado a la hora de aprender. Dado que es posible que los déficits observados en el uso de estrategias SRL estén vinculados, además de a otras condiciones, a déficits en el conocimiento sobre SRL, parece razonable disponer de instrumentos fiables y válidos que aporten información sobre dicho conocimiento.

Por todo ello, el objetivo principal de este estudio fue el análisis de las propiedades psicométricas (fiabilidad y validez) del Cuestionario de Evaluación del Conocimiento sobre Estrategias de Autorregulación (CEA), elaborado inicialmente por Rosário et al (2007) para adolescentes, en estudiantes universitarios. EI CEA fue desarrollado para la evaluación del conocimiento de los procesos autorregulatorios en estudiantes de enseñanza media, pero no se dispone de información sobre las propiedades psicométricas en estudiantes universitarios. Para el estudio de la validez predictiva se tendrán en cuenta los datos aportados por el Inventario de Autorregulación de Estrategias de Aprendizaje (IPPA) y los de la Escala de Evaluación de la Autorregulación del Aprendizaje a partir de Textos (ARATEX-R), así como las calificaciones académicas.

\section{MÉTODO}

\section{PARTICIPANTES}

En el presente estudio han participado 917 estudiantes de la Universidad de Oviedo, que cursaban sus titulaciones en las Facultades de Psicología y Logopedia ( $n=504 ; 55 \%$ ) o en la Facultad de Formación de Profesorado y Educación ( $n=413 ; 45 \%$ ). La mayoría de los estudiantes eran mujeres ( $n=770 ; 84 \%$ ), que asistían principalmente a los cursos de $1^{\circ}(n=$ $472 ; 51.5 \%), 2^{\circ}(n=126 ; 13.7 \%), 3^{\circ}$ ( $n=$ $319 ; 34.8 \%)$. La nota media de entrada en los estudios universitarios fue de 6.99, sobre 10 (mínimo 5, máximo 9,90), con una DT de .91. A nivel socio-educativo familiar, señalar que padres y madres tienen estudios principalmente secundarios (madres 39,4\%; padres $42,7 \%$ ) o universitarios (madres 34,3\%; padres 32,5\%); mientras que el $24 \%$ de las madres y el 22,6 de los padres solo tienen estudios a nivel primario.

\section{INSTRUMENTOS DE MEDIDA}

Además de la medida del conocimiento sobre estrategias SRL, tomada mediante el Cuestionario de Evaluación del Conocimiento sobre Estrategias de Autorregulación en universitarios (CEA-U), en el presente estudio se evaluó el uso de estas estrategias, a través del Inventario de Autorregulación 
de Estrategias de Aprendizaje (IPPA) y de la Escala de Evaluación de la Autorregulación del Aprendizaje a partir de Textos (ARATEX-R), así como el rendimiento académico de los estudiantes (mediante sus calificaciones).

- Cuestionario de Evaluación del Conocimiento sobre Estrategias de Autorregulación en universitarios (CEA-U). Con este cuestionario (adjunto en Apéndice A) se evaluó el nivel de conocimiento de los estudiantes sobre estrategias de autorregulación del aprendizaje. Está constituido de 10 cuestiones cerradas, con tres posibilidades de respuesta (dos falsas y una verdadera). Los ítems se refieren a diez estrategias fundamentales en el proceso autorregulatorio (Rosário, Mourão, Nuñez, González-Pienda, Solano, \&Valle, 2007), y que están asociadas en cuatro grupos: cognitivas, metacognitivas, motivacionales y de gestión de recursos. Dentro de las estrategias cognitivas, se preguntan cuestiones relacionadas con estrategias de toma de apuntes, elaboración y organización de la información y estrategias de preparación de exámenes. Respecto a las estrategias metacognitivas, se evalúan la planificación y la auto-evaluación. A nivel motivacional, se valora el aplazamiento de las tareas. Y en cuanto a las estrategias de gestión de recursos, se evalúan los conocimientos sobre organización y gestión del tiempo, así como la búsqueda de ayuda. Los datos de los estudios con alumnos adolescentes (pues no se dispone de datos con universitarios) indican una buena fiabilidad del cuestionario (alpha de Cronbach de .89) y una buena validez estructural (Rosário et al., 2007).

\section{- Inventario de Estrategias de} Autorregulación del Aprendizaje (IPPA). El IPPA ha sido elaborado por Rosário et al. (2007) y proporciona información sobre el uso de estrategias de autorregulación a la hora de aprender. Está basado en el modelo de B. J. Zimmerman (Zimmerman, 2011), en el que se identifican tres grandes fases: planificación, ejecución y evaluación. Conforme a esto, el IPAA está constituido por 12 elementos, cuatro ítems por fase, que se valoran en una escala de tipo Likert de 5 puntos (1-nunca a 5 -siempre). Se le pide al alumno que responda pensando en general y sobre la mayoría de sus asignaturas (eg., ítem 1: antes de comenzar a escribir un artículo, hago un plan: pienso en lo que voy a hacer y necesito para lograrlo). Los datos aportados por Cerezo et al. (2019) indican que el IPPA es fiable en términos de SRL (escala global), $\alpha=.78$ y $C R=.79$, aunque algo menor cuando atendemos a las tres dimensiones teóricas en particular, planificación: $\alpha=.47, \mathrm{CR}=.48$; evaluación: $\alpha=.56, \mathrm{CR}=.58$; ejecución: $\alpha=.62, \mathrm{CR}$ $=.62$ ), algo lógico si tenemos en cuenta el número reducido de ítems de cada factor. En relación a la validez, los resultados del análisis factorial confirmatorio de tres factores llevado a cabo por Cerezo et al. (2019) son satisfactorios, lo que aporta respaldo empírico en forma de validez de constructo del inventario.

- Escala de Evaluación de la Autorregulación del Aprendizaje a partir de Textos (ARATEX-R). Esta escala proporciona información sobre el uso de estrategias SRL cuando los estudiantes están aprendiendo de textos. Los estudios de validez con estudiantes universitarios muestran que ARATEX-R dispone de cinco dimensiones: planificación, cognición, motivación, evaluación y gestión del contexto (Núñez et al., 2015). La escala comienza con una declaración general: «cuando estoy estudiando o trabajando en un texto (...)». Los estudiantes deben valorar en qué medida funcionan dentro de ese contexto como se indica en los 20 ítems. La respuesta se ofrece en una escala tipo Likert de 5 puntos (1 -nunca a 5 -siempre). La fiabilidad de la escala global es buena ( $\alpha=.89, C R=.92)$, y lógicamente algo menor para los cinco factores mencionados (motivación: $\alpha=.78, \mathrm{CR}=.78$; planificación: $\alpha=.84, C R=.82$; gestión del contexto: $\alpha=$ $.45, \mathrm{CR}=.45$; cognición: $\alpha=.88, \mathrm{CR}=.89$; evaluación: $\alpha=.70, C R=.71$ ), especialmente en el factor de gestión del contexto ya que solo tiene dos elementos.

- Rendimiento académico. Se tienen en cuenta dos medidas de rendimiento: previo y posterior. Por un lado, la nota 
de entrada a la Universidad (y que por ser anterior a la evaluación de las variables SRL denominamos «rendimiento previo»). Por otro, las calificaciones finales de los estudiantes en el curso académico en que se realizó la evaluación y la intervención fueron utilizadas como medida del rendimiento «académico posterior» (y que aquí denominamos así por ser posterior a la intervención y las medidas de las variables).

\section{PROCEDIMIENTO}

Los estudiantes que constituyen la muestra descrita han participado en un programa de mejora de estrategias de autorregulación, el cual ha tenido una duración de un trimestre (de septiembre a diciembre). Las escalas de evaluación utilizadas para este estudio se aplicaron en dos ocasiones: antes y después de la intervención. De este modo, se dispone de medidas repetidas para los estudios de validez y fiabilidad.

\section{ANÁLISIS DE DATOS}

Los datos han sido analizados en tres fases. Inicialmente, se aportan datos descriptivos de la escala CEA-U (correlaciones de Pearson de dos colas, medias, desviaciones típicas, asimetría y curtosis). Posteriormente, con ayuda del programa AMOS 22 (Arbuckle, 2013), se estudia la validez de constructo de la escala mediante análisis factorial confirmatorio (AFC), tomando en cuenta las dos medidas, atendiendo tanto al ajuste del modelo como a su invarianza. La evaluación del modelo factorial se realiza tanto en base al valor de chi-cuadrado $\left(\chi^{2}\right)$ y su probabilidad asociada (p), como también a partir de la información proporcionada por índices de ajuste como el SRMR, GFI y AGFI (Jöreskog \& Sörbom, 1983), el CFI (Bentler, 1990), y el RMSEA (Browne \& Cudeck, 1993). El modelo ajusta bien cuando $\mathrm{GFI}$ y $\mathrm{AGFI}>.90, \mathrm{CFI}$ $>$.95, y SRMR y RMSEA $\leq .05$. Se utilizan también los criterios de información de Akaike (AIC) y el bayesiano de Schwarz (BIC) para la comparación de modelos. Finalmente, se analiza la fiabilidad de la escala, tanto por el procedimiento de consistencia interna como test-retest.

\section{RESULTADOS}

\section{ESTADÍSTICOS DESCRIPTIVOS}

En la Tabla 1 se aportan los estadísticos descriptivos correspondientes a los ítems del CEA-U (matriz de correlaciones, medias, y desviaciones típicas) en los dos momentos de medida. La prueba de esfericidad de Batlett muestra que los diez ítems están significativamente correlacionados, tanto en la primera medida $\left(\chi^{2}(45)=833.56, p<\right.$ $.001)$ como en la segunda medida $\left(\chi^{2}(45)=\right.$ 725.70, $p<.001)$.

\section{VALIDEZ}

Se han calculado dos tipos de validez de la escala CEA-U: (a) de constructo (mediante análisis factoriales confirmatorios), y (b) predictiva (por su asociación con el uso de estrategias autorregulatorias a la hora de aprender y el rendimiento).

\section{VALIDEZ DE CONSTRUCTO}

Tal como se indicó, esta escala pretende aportar información sobre los conocimiento que poseen los estudiantes en relación a cuatro tipos de estrategias de aprendizaje autorregulado (cognitivas, metacognitivas, motivacionales y de gestión de recursos). No obstante, los propios autores, Rosário et al. (2007), en base a datos aportados por estudiantes de educación obligatoria, informan que se trata de una escala unifactorial, al menos a estas edades. Sin embargo, no hay información sobre la estructura de esta escala con estudiantes universitarios.

En este estudio se contrastan dos modelos: unifactorial y multifactoral. El modelo unifactorial está justificado por los datos aportados por los estudios previos (con muestras de enseñanza no universitaria). El modelo multifactorial (de cuatro factores según proponen los autores originalmente y que ha sido descrito en el apartado de instrumentos) 
Tabla 1

Estadísticos descriptivos del CEA-U en la primera y segunda medida.

\begin{tabular}{|c|c|c|c|c|c|c|c|c|c|c|}
\hline & 1 & 2 & 3 & 4 & 5 & 6 & 7 & 8 & 9 & 10 \\
\hline \multicolumn{11}{|c|}{ Primera medida } \\
\hline 1 & - & & & & & & & & & \\
\hline 2 & $.124^{\star \star}$ & - & & & & & & & & \\
\hline 3 & $.143^{* *}$ & $.130^{* *}$ & - & & & & & & & \\
\hline 4 & $.124^{\star \star}$ & $.186^{\star *}$ & $.098^{* *}$ & - & & & & & & \\
\hline 5 & .052 & $.188^{* *}$ & .012 & $.303^{* *}$ & - & & & & & \\
\hline 6 & $.246 * \star$ & $.179 * *$ & $.135^{* *}$ & $.120 * *$ & $.085^{\star *}$ & - & & & & \\
\hline 7 & $.195^{\star \star}$ & $.145^{* *}$ & $.100^{* *}$ & $.236 * \star$ & $.170^{\star \star}$ & $.215^{\star \star}$ & - & & & \\
\hline 8 & $.169 * *$ & $.100^{* *}$ & $.132^{* *}$ & $.182^{\star *}$ & $.142^{\star \star}$ & $.137^{\star *}$ & $.196^{* *}$ & - & & \\
\hline 9 & $.107 \star \star$ & $.247^{* *}$ & $.097^{* *}$ & $.193^{\star \star}$ & $.247^{* \star}$ & $.097 \star \star$ & $.123^{\star \star}$ & $.127^{* *}$ & - & \\
\hline 10 & $.155^{\star \star}$ & $.215^{* *}$ & $.151^{* *}$ & $.288^{\star *}$ & $.284^{* *}$ & $.193^{\star \star}$ & $.264^{\star \star}$ & $.170^{\star *}$ & $.281^{* *}$ & - \\
\hline M & 0.68 & 0.72 & 0.83 & 0.86 & 0.93 & 0.66 & 0.89 & 0.95 & 0.87 & 0.81 \\
\hline DT & 0.47 & 0.45 & 0.38 & 0.34 & 0.26 & 0.47 & 0.32 & 0.21 & 0.34 & 0.39 \\
\hline \multicolumn{11}{|c|}{ Segunda medida } \\
\hline 1 & - & & & & & & & & & \\
\hline 2 & $.183^{\star \star}$ & - & & & & & & & & \\
\hline 3 & $.134^{\star \star}$ & $.121^{* *}$ & - & & & & & & & \\
\hline 4 & .251 ** & $.125^{* *}$ & $.118^{* *}$ & - & & & & & & \\
\hline 5 & $.182^{\star \star}$ & $.121^{* *}$ & .026 & $.077^{*}$ & - & & & & & \\
\hline 6 & $.210^{* *}$ & $.176^{* *}$ & $.130^{* *}$ & $.166^{* *}$ & $.200^{\star *}$ & - & & & & \\
\hline 7 & $.182^{\star *}$ & $.084^{*}$ & .006 & $.173^{* *}$ & $.179^{* *}$ & $.209 * *$ & - & & & \\
\hline 8 & $.156^{\star \star}$ & .063 & .025 & $.109 * *$ & .057 & $.227^{\star \star}$ & $.158^{\star \star}$ & - & & \\
\hline 9 & $.120^{\star \star}$ & $.194^{* *}$ & .042 & $.179 * *$ & $.084^{*}$ & $.227^{\star \star}$ & $.171^{* *}$ & $.154^{* *}$ & - & \\
\hline 10 & $.204^{\star \star}$ & $.182^{\star *}$ & $.086^{* *}$ & $.204^{\star \star}$ & .143 & $.218^{\star \star}$ & $.235^{\star \star}$ & $.209^{* *}$ & $.233^{\star *}$ & - \\
\hline$M$ & 0.77 & 0.78 & 0.83 & 0.90 & 0.95 & 0.77 & 0.92 & 0.96 & 0.90 & 0.85 \\
\hline DT & 0.42 & 0.41 & 0.37 & 0.31 & 0.21 & 0.42 & 0.27 & 0.20 & 0.30 & 0.45 \\
\hline
\end{tabular}

se justifica porque los estudiantes universitarios disponen de mayores competencias y experiencia que los estudiantes de secundaria y, como consecuencia, podrían realizar una mayor diferenciación del constructo.

Los datos de ajuste del modelo unifactorial aportados por los estudiantes en ambas medidas, en general, muestran un ajuste aceptable: primera medida $\left(\chi^{2}(35)=118.39\right.$; $p<.001 ;$ SRMR $=.006 ; \mathrm{GFI}=.97 ; \mathrm{AGFI}=$ $.96 ; \mathrm{CFI}=.90 ; \mathrm{RMSEA}=.051$ (.041-.061); $\mathrm{AIC}=158.38 ; \mathrm{BIC}=254.81)$, segunda medida $\left(\chi^{2}(35)=75.48 ; p<.001 ;\right.$ SRMR $=.004 ; \mathrm{GFI}=.98 ; \mathrm{AGFI}=.98 ; \mathrm{CFI}=.95 ;$ RMSEA $=.036(.024-.047) ; \mathrm{AIC}=115.48$; $\mathrm{BIC}=211.90)$. Al comparar los datos de ambas muestras, se observa que el ajuste del modelo en la segunda medida es óptimo, y mejor que el aportado por los datos de la primera medida (AIC y BIC son menores en la segunda medida, y los índices de ajuste mejores). Por otra parte, el ajuste del modelo multidimensional de cuatro factores no ha sido posible por falta de convergencia. 


\section{VALIDEZ PREDICTIVA}

La validez predictiva del CEA-U se estudió examinando las correlaciones entre esta medida y otras con las que debería estar asociada, tales como el uso de estrategias de autorregulación (generales y específicas a la comprensión de textos) y el rendimiento académico (tanto pasado como futuro). En términos generales, se hipotetizó que: (a) si bien un buen conocimiento de estrategias SRL no necesariamente tiene que llevar a su uso, en principio si debería observarse una relación positiva y estadísticamente significativa; y (b) si ocurre lo anterior, entonces, deberíamos obtener una relación positiva y estadísticamente significativa entre conocimiento en estrategias SRL y rendimiento académico. En la Tabla 2 se aportan los datos obtenidos del estudio correspondientes a estas dos hipótesis.
En términos generales, los resultados obtenidos confirman las hipótesis formuladas. En concreto, atendiendo a la primera columna de la Tabla 2, se observa que cuanto mayor es el conocimiento en SRL mayor es el uso de estrategias SRL, tanto a nivel general como en el contexto específico de la comprensión de textos. Asimismo, un mayor conocimiento de SRL también está positivamente asociado con el rendimiento académico, tanto previo como posterior.

\section{FIABILIDAD}

Obtenida evidencia empírica de la unidimensionalidad factorial del CEA-U, se procedió al cálculo de la fiabilidad general de la escala, en los dos momentos de la aplicación, mediante el estadístico alpha de Cronbach y el índice de fiabilidad compuesta (IFC). Los datos obtenidos muestran valores moderados de fiabilidad en ambos momentos

Tabla 2

Relación entre conocimiento en SRL, uso de estrategias SRL y rendimiento académico.

\begin{tabular}{|c|c|c|c|c|c|}
\hline & 1 & 2 & 3 & 4 & 5 \\
\hline \multicolumn{6}{|l|}{ Primera medida (previa) } \\
\hline 1. Conocimiento en SRL & - & & & & \\
\hline 2. Uso de SRL general (IPPA) & $.256 * *$ & - & & & \\
\hline 3. Uso de SRL específico (ARATEX-R) & $.226 * *$ & $.675^{\star *}$ & - & & \\
\hline 4. Rendimiento previo & $.182^{\star \star}$ & $.132 * *$ & $.103^{* *}$ & - & \\
\hline 5. Rendimiento posterior & $.157^{\star \star}$ & $.197^{\star *}$ & $.143^{* *}$ & $.240 * *$ & - \\
\hline \multicolumn{6}{|l|}{ Segunda medida (posterior) } \\
\hline 1. Conocimiento en SRL & - & & & & \\
\hline 2. Uso de SRL, general (IPPA) & $.276^{* *}$ & - & & & \\
\hline 3. Uso de SRL específico (ARATEX-R) & $.282^{\star \star}$ & $.683^{* *}$ & - & & \\
\hline 4. Rendimiento previo & $.110 * *$ & $.118^{* *}$ & .037 & - & \\
\hline 5. Rendimiento posterior & $.188^{* *}$ & $.191^{\star *}$ & $.171^{\star *}$ & $.464^{* *}$ & - \\
\hline
\end{tabular}


de medida: previo a la intervención $(\alpha=.67$; IFC $=.68$ ) y posterior a la intervención ( $\alpha=$ .65 ; IFC $=.67$ ). Por otra lado, los datos de la fiabilidad por el procedimiento test-retest muestran una buena consistencia temporal: $r_{1.2}=.561, p<.001, d=1.36$ (con un tamaño del efecto grande).

\section{DISCUSIÓN}

SRL hace referencia al modo en que los estudiantes controlan el aprendizaje de manera proactiva y cómo manejan sus procesos cognitivos y motivacionales hacia sus objetivos auto-establecidos (Zimmerman, 2008). Investigaciones previas, ofrecen un sólido corpus de datos empíricos que indican una fuerte relación entre el uso de estrategias SRL y el éxito académico (e.g., Boekaerts \& Corno, 2005; Liem, Lau, \& Nie, 2008; Núñez, Cerezo, González-Pienda, Rosário, Valle, Fernández, \& Suárez, 2011 ; Rosário, Núñez, González-Pienda, Valle, Trigo, \& Guimarães, 2010; Zimmerman \& Martínez-Pons, 1988). Por ello, entre otros aspectos, en las últimas décadas se han llevado a cabo numerosos intentos por dotar a los estudiantes con conocimientos y habilidades que les permitan autorregular su aprendizaje de diferentes maneras (Cleary \& Zimmerman, 2004; Dignath et al., 2008). Esta capacitación en SRL ayuda a los estudiantes a comprender la importancia y la instrumentalidad del uso de estrategias motivacionales, conductuales y metacognitivas para controlar su aprendizaje (Weinstein, Husman \& Dierking, 2000). Esta suposición resalta varios aspectos esenciales del SRL: el conocimiento y uso de estrategias de SRL por parte de los estudiantes, la percepción de autoeficacia, la utilidad percibida, y el compromiso personal con los objetivos educativos.

Aunque existe un buen número instrumentos de evaluación, principalmente de tipo autoinforme, que aportan información sobre en qué medida los estudiantes utilizan estrategias necesarias para la autorregulación del aprendizaje $y$, por tanto, para un aprendizaje autónomo, no disponemos de instrumentos fiables y teóricamente validos que aporten información sobre el grado de conocimiento del estudiante sobre lo que implica un comportamiento autorregulado a la hora de aprender. Esta ausencia de instrumentos quizás sea debida a que se da por hecho que tal conocimiento ya forma parte de la memoria a largo plazo de los estudiantes, y más cuanto mayores son éstos. No obstante, esto no es así en un buen porcentaje de alumnos (Rosário et al., 2013). Lo anterior, sumado al hecho de que podría ser que los déficits observados en el uso de estrategias autoreguladoras a la hora de aprender estén vinculados, en cierta medida, a déficits en el conocimiento sobre SRL, es lo que ha motivado principalmente el estudio de las propiedades psicométricas del CEA-U.

En base a un diseño de medidas repetidas (dos medidas con un intervalo temporal de seis meses) se ha aplicado el CEA-U a una amplia muestra de estudiantes de las titulaciones de psicología y educación. Se estudió la validez y la fiabilidad de la escala por una doble vía: la validez estructural y la predictiva; así como la fiabilidad por consistencia interna y testretest.

Los resultados obtenidos muestran al CEA-U como un instrumento válido y fiable para su utilización con estudiantes universitarios. En concreto, se obtuvo evidencia empírica de la unidimensionalidad propuesta por Rosário et al. (2007) y una excelente consistencia en la capacidad predictiva tanto del uso de estrategias de autorregulación como del rendimiento académico. Las correlaciones halladas indicaron que aquellos alumnos que disponen de más conocimiento sobre las estrategias específicas de la autorregulación del aprendizaje más utilizan éstas en su día a día (con un tamaño del efecto importante: $d=.57$ en estrategias SRL generales; y $d=.59$ en estrategias SRL específicas en comprensión de textos), como estudiantes y mejor rendimiento académico obtienen (d $=.38$ para la predicción del rendimiento). Obviamente es menor el tamaño del efecto en la predicción del rendimiento pues el conocimiento en estrategias SRL está más cercana al uso de tales estrategias de lo que lo está del rendimiento académico (ya que éste 
depende de muchísimas más variables que del conocimiento en SRL). No obstante, habría que tomar en cuenta que los tamaños de los efectos no son grandes ni para la predicción del rendimiento académico ni para la predicción del uso de estrategias SRL. Quizás esto esté relacionado con que no siempre los procesos de aprendizaje, y el rendimiento, está directamente relacionados con el uso de tales estrategias. Es posible que aún tengan lugar procesos de aprendizaje demasiado dirigidos por los procesos de enseñanza, con escaso margen a la autonomía.

Con todo, los resultados derivados del CEA- $U$ deben utilizarse con cierta cautela pues existen dos aspectos problemáticos. En primer lugar, está el hecho de que a nivel de estudios superiores el estudiante, por lo general, ya dispone de un buen conocimiento sobre estrategias SRL y, por tanto, es posible que la escala de medida actual conlleve cierta asimetría y kurtosis. Por ello, sería de interés que estudios posteriores tuvieran como objetivo la mejora de la escala de medida de la versión actual del CEA-U (acierto-fallo), por ejemplo, utilizando una escala continua (o al menos de tipo ordinal) que pueda captar diferentes modos y grados de entender el aprendizaje autorregulado y el uso específico de las estrategias implicadas.

En segundo lugar, existe un evidente problema de fiabilidad en términos de consistencia interna de la escala, aunque no en relación a su estabilidad temporal. Como se ha podido comprobar por los datos del alpha de Cronbach y por el índice de fiabilidad compuesta, la consistencia de la escala, con ser aceptable, puede mejorar significativamente. Consecuentemente, sería de interés refinar la escala en su aplicación con universitarios para mejorar su consistencia interna. Por ejemplo, aunque hoy en día se tiende al uso de instrumentos breves, una posible vía para el incremento de la fiabilidad de esta escala es el incremento del número de ítems (principalmente respecto de la evaluación de ña metacognición, de la gestión de recursos y, fundamentalmente, respecto de las estrategias motivacionales y afectivas).
Con todo, tal como se ha indicado, CEA-U es un instrumento suficientemente fiable y válido para la evaluación del conocimiento de estrategias de autorregulación del aprendizaje en contextos de Educación Superior.

\section{- Agradecimientos}

Este trabajo ha sido financiado por el Ministerio de Ciencia e Innovación (EDU2010-16231), el Ministerio de Economía y Competitividad - Dirección General de Investigación Científica y Técnica (EDU2014-57571-P) y el Principado de Asturias (FC-GRUPIN-IDI/2018/000199).

\section{- Conflicto de intereses.}

Los autores declaran no tener ningún conflicto de intereses.

\section{REFERENCIAS}

Allgood, W. P., Risko, V. J., Álvarez, M. C. \& Fairbanks, M. M. (2000). Factors that influence study. En R. F. Flippo y D. C. Caverly (Coord.), Handbook of college reading and study strategy research, (pp. 201-219). NJ: LEA.

Arbuckle, J. L. (2013). Amos 22.0 User's Guide. Crawfordville: Amos Development Corporation.

Bandura, A. (2001). Social cognitive theory: An genetic perspective. American Review of Psychology, 52, 1-26. doi:10.1146/annurev. psych.52.1.1

Bentler, P. M. (1990). Comparative fit indexes in structural models. Psychological Bulletin, 107, 238-246. Doi: 10.1037/00332909.107.2.238

Biggs,J.(2001). "Thereflectiveinstitution: Assuring and enhancing the quality of teaching and learning". Higher Education, 42, 221-237. doi.org/10.1080/0260293960210101

Boekaerts, M., \& Corno, L. (2005). Selfregulation in the classroom: A perspective on assessment and intervention. Applied Psychology: An International Review, 54, 199- 231. doi.org/10.1111/i.14640597.2005.00205.x

Browne, M. W., \& Cudeck, R. (1993). Alternative ways of assessing model fit. In K. Bollen \& J. Long (Eds.), Testing Structural Equation Models (pp. 136-162). Newbury Park, CA: Sage. doi:10.1111/i.1600-0047.2004.00316.x 
Cano, F. (2005). Consonance and dissonance in students' learning experience. Learning and Instruction, 15, 201-223. doi: 10.1016/i. learninstruc.2005.04.003

Cerezo, R., Fernández, E., Amieiro, N., Valle, A., Rosário, P., \& Núñez, J. C. (2019). Mediating role of self-efficacy and usefulness between self-regulated learning strategy knowledge and its use. Revista de Psicodidáctica, 24 (1), $1-8$.

Cerezo, R., Sánchez-Santillán, M., Paule, M. P., \& Núñez, J. C. (2016). Students' LMS interaction patterns and their relationship with achievement: Acase study in higher education. Computers \& Education, 96, 42-54. doi. org/10.1016/i.compedu.2016.02.006

Cheung, C. K. \& Kwok, S. T. (1998). Activities and academic achievement among college students. The Journal of Genetic Psychology, 159 (2), 147 - 162. doi: 10.1080/00221329809596142

Cleary, T. J., \& Zimmerman, B. J. (2004). Selfregulation empowerment program: A schoolbased program to enhance self-regulated and self-motivated cycles of student learning. Psychology in the Schools, 41, 537-550. doi: 10.12691/education-2-11A-2

Dignath, Ch., Buettner, G., \& Langfeldt, H. P. (2008). How Can Primary School Students Learn Self-Regulated Learning Strategies Most Effectively? A Meta-Analysis on SelfRegulation Training Programmes. Educational Research Review, 3(2), $101-129$.

Entwistle, N., \&Waterston, S. (1988). Approaches to studying and levels of processing in university students. British Journal of Educational Psychology, 58, 258 - 265. doi: doi.org/10.1111/i.2044-8279.1988. tb00901.x

Garavalia, L. S. \& Gredler, M. E. (2002). Prior achievement aptitude and use of learning strategies as predictors of college student achievement. College Student Journal, 36, 616-626. doi.org/10.4219/jaa-2008-775

González, J. \& Wagenaar, R. (Eds.) (2003). Tuning Educational Structures in Europe. Informe final. Fase Uno. Bilbao: Universidad de Deusto.
Jöreskog, K. G., \& Sörbom, D. (1983). LISREL - 6 User's reference guide. Mooresville, IN: Scientific Software.

Liem, A. D., Lau, S., \& Nie, Y. (2008). The role of self-efficacy, task value, and achievement goals in predicting learning strategies, task disengagement, peer relationship, and achievement outcome. Contemporary Educational Psychology, 33, 486-512. doi:10.12691/education-1-3-4

Nota, L., Soresi, S. \& Zimmerman, B. J. (2005). Self-regulation and academia and resilience: a longitudinal study. International Journal of Educational Research, 41, 198-251.

Núñez, J. C., Amieiro, N. Álvarez, D., García, T., \& Dobarro, A. (2015). Escala de Evaluación de la Autorregulación del Aprendizaje a partir de Textos (ARATEX-R). European Journal of Education and Psychology, 8, 9-22.

Núñez, J. C., Cerezo, R., González-Pienda, J. A., Rosário, P., Valle, A., Fernández, E., \& Suárez, N. (2011). Implementation of training programs in self-regulated learning strategies in Moodle format: Results of an experience in higher education. Psicothema, 23, 274-281.

Núñez, J. C., González-Pienda, J., Solano, P. \& Rosário, P. (2006). Evaluación de los procesos de autorregulación mediante autoinforme. Psicothema, 18, 353-358.

Paunonen, S. V. \& Ashton, M. C. (2001). Big five predictors of academic achievement. Journal of Research in Personality, 35, 7890. doi:10.1006/irpe.2000.2309

Pike, G. R. \& Kuh, G. (2005). A typology of student engagement for American colleges and universities. Research in Higher Education, 46, 185-209. doi:10.1007/s $11162-004-1599-0$

Pintrich, P. R. (2000). The role of goal orientation in self-regulated learning. En M. Boekaerts, P.R. Pintrich, y M. Zeidner (Eds.), Handbook of self-regulation, (pp. 452-502). San Diego, California: Academic Press.

Pintrich, P. R. (2004). A conceptual framework for assessing motivation and self-regulated learning in college students. Educational Psychology Review, 16, 385-407. doi. 


\section{org/10.1007/s10648-004-0006-x}

Pintrich, P. R. \& De Groot, E. (1990). Motivational and self-regulated learning components of classroom academic performance. Journal of Educational Psychology, 82, 33-40. doi. org/10.1037/0022-0663.82.1.33

Plant, E. A., Ericsson, K. A., Hill, L. \& Asberg, K. (2005). Why study time does not predict grade point average across college students: implications of deliberate practice for academic performance. Contemporary Educational Psychology, 30, 96-116. doi. org/10.1016/i.cedpsych.2004.06.001

Rosário, P., González-Pienda, J. A., Pinto, R., Ferreira, P., Lourenço, A. \& Paiva, O. (2010). Efficacy of the program "Testas's (mis)adventures" to promote the deep approach to learning. Psicothema, 22, 828-834.

Rosário, P., Mourão, R., Nuñez, J. C., González-Pienda, J., Solano, P., \& Valle, A. (2007). Evaluating the efficacy of a program to enhance college students' self-regulation learning processes and learning strategies. Psicothema, 19, 422427.

Rosário, P., Mourao, R., Soares, S., Chaleta, E., Grácio, L., Simoes, F., Núñez, J. C., \& González-Pienda, J. A. (2005). Trabalho de casa, tarefas escolares, auto-regulaçao e envolvimento parental. Psicología em Estudo, 10, 343-351.

Rosário, P., Núñez, J. C., González-Pienda, J. A., Valle, A., Trigo, L., \& Guimarães, C. (2010). Enhancing self-regulation and approaches to learning in first-year college students: A narrative-based program assessed in the lberian Peninsula. European Journal of Psychology of Education, 25, $411-428$.

Rosário, P., Núñez, J. C., González-Pienda, J.A., Almeida, L., Soares, S. \& Rubio, M. (2005). El aprendizaje escolar examinado desde la perspectiva del «Modelo 3P» de J. Biggs. Psicothema, 17, 20-30.

Rosário, P., Núñez, J. C., Valle, A., GonzálezPienda, J. A., \& Lourenço, A. (2013). Grade level, study time, and grade retention and their effects on motivation, self-regulated learning strategies, and mathematics achievement: a structural equation model. European Journal of Psychology of Education, 28, 1311-1331. doi:10.1007/s10212-012-0167-9

Tuckman, B. W. (2003). The effect of learning and motivation strategies training on college students' achievement. Journal of College Student Development, 44, 430437. doi.org/10.1353/csd.2003.0034

Weinstein, C. E., Husman, J., \& Dierking, D. (2000). Self-regulation intervention with a focus on learning strategies. In M. Boekaerts, P. Pintrich, \& M. Zeidner (Eds.), Handbook of self-regulation (pp. 727-747). New York, San Diego: Academic press.

Williams, J.E. \& Hellman, C.M. (1998). Investigating self-regulated learning among first-generation community college students. Journal of Applied Research in the Community College, 5, 83-87.

Williams, P. E. \& Hellman, C. M. (2004). Differences in self-regulation for online learning between first-and second-generation college students. Research in Higher Education, 45, 71 - 82.

Winne, P. H. \& Jamieson-Noel, D. (2002). Exploring students' calibration of selfreports about study tactics and achievement. Contemporary Educational Psychology, 27, 551-572. doi.org/10.1016/S0361476X(02)00006-1

Winne, P. H. \& Jamieson-Noel, D. (2003). Selfregulating studying by objectives for learning: Students' reports compared to a model. Contemporary Educational Psychology, 28, 259-276. doi.org/10.1016/S0361 476X(02)00041-3

Winne, P. H. \& Perry, N. E. (2000). Measuring self-regulated learning. En M. Boekaerts, P.R. Pintrich y M. Zeidner (Eds.), Handbook of self - regulation ( pp. 531 -566). NY.: Academic Press.

Wolters, C. A. (2003). Regulation of motivation: Evaluating an underemphasized aspect of self-regulated learning. Educational Psychologist, 38, 189-205. doi.org/10.1207/ 


\section{S15326985EP3804 1}

Zimmerman, B. J. (1998). Developing selffulfilling cycles of academic regulation: An analysis of exemplary instructional models. En D. H. Schunk y B. J. Zimmerman (Eds.), Self-Regulated learning. From teaching to Self-Reflective Practice (pp.1-19). Hillsdale, NJ: Lawrence Erlbaum Associates, Inc.

Zimmerman, B. J. (2002). Becoming a selfregulated learner: An overview. Theory into Practice, 41, 64-70. doi.org/10.1207/ s15430421 tip4102 2

Zimmerman, B. J. (2008). Investigating selfregulation and motivation: Historical, background, methodological developments, and future prospects. American Educational Research Journal, 45, 166-183. doi. org/10.3102/0002831207312909

Zimmerman, B. J. (2011). Motivational sources and outcomes of self-regulated learning and performance. In B. J. Zimmerman \& D. H. Schunk (Eds.), Handbook of self-regulation of learning and performance (pp. 49-65). NY: Routledge.

Zimmerman, B. J. \& Bandura, A. (1994). Impact of self-regulatory influences on writing course attainment. American Educational Research Journal, 31, 845-862. doi. org/10.2307/1163397

Zimmerman, B. J., \& Martinez-Pons, M. (1988). Construct validation of a strategy model of student self-regulated learning. Journal of Educational Psychology, 80, 284-290. doi. org/10.1037/0022-0663.80.3.284

\section{APÉNDICE A.}

\section{CUESTIONARIO DE EVALUACIÓN DEL CONOCIMIENTO SOBRE ESTRATEGIAS DE AUTORREGULACIÓN EN UNIVERSITARIOS (CEA-U)}

Responde a este cuestionario, eligiendo la opción que consideres más correcta (sólo una):

1. Antes de comenzar a hacer cualquier tarea (examen, trabajo,...) es importante:

a. Pensar en los objetivos y metas, y hacer un plan para conseguirlos contando con los recursos personales... b. Esperar a que el profesor diga lo que hay que hacer.

c. Ponerse a la tarea sin perder tiempo evitando excusas.

2. Un aspecto fundamental en el estudio personal es la organización y gestión del tiempo, lo cual implica:

a. Preparar horarios para organizar el tiempo en la semana antes de los exámenes.

b. Hacer horarios personales que incluyan tiempo de estudio diario, preparación de exámenes, de trabajos, ocio...

c. Ser flexibles en la realización de las tareas, dejando espacio para la improvisación, y huir de la rigidez y del estrés.

3. Para evitar el aplazamiento (dejar las tareas de estudio para otro momento) lo mejor es:

a. Prometerse recompensas si se consigue acabar la tarea a tiempo.

b. Dividir la tarea en pequeñas metas y organizar el tiempo para cada una.

c. Intentar motivarse haciendo otras cosas (ir a tomar un café, chatear...) y ponerse después con la tarea.

4. Para tomar apuntes que ayuden a la hora de estudiar y preparar los exámenes, es importante:

a. Intentar recoger literalmente todo lo que dice el profesor.

b. Fotocopiar los apuntes del mejor estudiante de la clase.

c. Anotar los aspectos más importantes y completarlos en casa con otras informaciones.

5. Subrayar es una estrategia de estudio cuya función principal es:

a. Señalar las partes de los contenidos que después se deberán estudiar.

b. Seleccionar la información más importante después de leer y comprender el texto.

c. Decorar los apuntes para hacerlos más amenos y motivadores a la hora de estudiar.

6. Los resúmenes y mapas conceptuales tienen como objetivo:

a. Organizar y elaborar de forma personal la información, jerarquizándola según su nivel de importancia.

b. Reducir la información facilitando la tarea de estudiar.

c. Escribir la información para memorizarla. 
7. Para aprender y estudiar un texto, es importante memorizar de forma comprensiva, lo que implica:

a. Re-escribir los contenidos para aprenderlos.

b. Relacionar la nueva información con los conocimientos que ya se poseen buscando conexiones entre ellos.

c. Repetir los nuevos contenidos una y otra vez hasta saberlos de memoria.

8. En la preparación de los exámenes, se debe:

a. Estudiar la noche antes del examen para asegurar el recuerdo.

b. Usar chuletas para datos y fechas que no tiene sentido aprender de memoria.

c. Tener en cuenta el tipo de examen, ya que las estrategias de estudio se deben adecuar al mismo.
9. En el estudio personal, la búsqueda de ayuda ante una dificultad se considera:

a. Una manera constructiva y muy importante de resolver problemas cuando uno solo no puede.

b. Una estrategia útil para evitar un potencial fracaso.

c. Una forma de "tirar la toalla" y desistir.

10. Después de realizar un examen, trabajo... se debe:

a. No hacer nada, porque lo importante ya se ha hecho, sólo queda esperar la nota.

b. Analizar lo que se ha hecho y los resultados, para sacar conclusiones y mejorar.

c. Valorar los resultados obtenidos en comparación con los compañeros. 